\title{
Boosting clinical translation of nanomedicine
}

\author{
"Biomimetic platforms, such as organs-on-a-chip, will \\ provide unprecedented opportunities to accurately \\ evaluate the safety and efficacy of nanomedicine \\ formulations, leading to higher clinical trial success rates."
}

First draft submitted: 27 March 2016; Accepted for publication: 5 April 2016; Published
online: 13 May 2016

Keywords: artificial organ • clinical translation $\bullet$ nanobiomaterials $\bullet$ nanomedicine

- organs-on-a-chip

In the past few decades, the field of nanotechnology has significantly progressed, leading to success in the design and synthesis of functional nanoparticles for therapeutic and diagnostic applications. Nanomedicine, which is the application of nanotechnology in medicine, can be utilized to create novel formulations for targeted drug delivery that are potentially safer and thus can lead to better patient outcomes through a reduction in drug side effects. However, the clinical translation of these formulations has been limited due to expensive and lengthy regulatory processes and the inability to comprehensively assess the safety and efficacy of the nanomedicines. Thus, rigorous and innovative preclinical testing models are needed to evaluate these formulations to ensure their successful clinical translation and future commercial availability.

\section{Nanomedicine at play}

The unique physiochemical properties of nanomaterials, endowed by their nanometer scale, have enabled new mechanisms to diagnose and treat diseases with enhanced efficacy through maximized target specificity [1]. For example, nanoparticles can be used as therapeutic carriers to encapsulate and release drugs at target sites via systemic delivery and high accessibility to region where large-sized particles cannot reach [2]. Also, nanoparticles containing contrast agents can act as diagnostic sensors for real-time detection and visual- ization of diseases inside the body [3]. In addition, nanoparticles with optimized shapes and sizes can modulate cellular functions through direct attachment onto the surface of or internalization by the cells [4]. Furthermore, biomimetic nanoparticles have facilitated tissue regeneration through enhanced host integration $[5,6]$. However, despite the rapid advancement of nanotechnologies in biomedicine, the translation into practice has been relatively slow.

\section{Clinical \& regulatory limitations for nanomedicine translation}

The main reason for the slow rates of clinical translation lies in the extremely high cost and lengthy time period associated with clinical trials, largely caused by a lack of proper preclinical evaluation platforms that can accurately predict the performance of nanomedicine in the human body during the preclinical phases. As a result, although pharmaceutical companies have aimed to bring nanoparticlebased therapeutics into the market due to their high treatment efficacy, the failure and drug attrition rates still remain high during both the clinical trials and after-market entry.

Thus far, only about 175 nanomedicine products have been commercialized, although a significant amount of formulations have been researched [7,8]. In the United States, it usually takes $8-20$ years for nanomedicine development and preclinical investigations, including in vitro cell studies and
Hae Lin Jang ${ }^{1,2,3}$, Yu Shrike Zhang ${ }^{1,2,3}$ \& Ali Khademhosseini ${ }^{* 1,2,3,4,5}$ 'Biomaterials Innovation Research Center, Division of Biomedical Engineering, Department of Medicine, Brigham \& Women's Hospital, Harvard Medical School, Boston, MA 02139, USA ${ }^{2}$ Harvard-Massachusetts Institute of Technology Division of Health Sciences \& Technology, Massachusetts Institute of Technology, Cambridge, MA 02139, USA ${ }^{3}$ Wyss Institute for Biologically Inspired Engineering, Harvard University, Boston, MA 02115, USA

${ }^{4}$ Department of Bioindustrial Technologies, College of Animal Bioscience \& Technology, Konkuk University, Hwayang-dong, Gwangjin-gu, Seoul 143-701, Republic of Korea ${ }^{5}$ Department of Physics, King Abdulaziz University, Jeddah 21569, Saudi Arabia *Author for correspondence: alik@bwh.harvard.edu 
in vivo animal tests; these results are subsequently submitted to the Food and Drug Administration (FDA) to obtain permission to conduct human clinical trials, which takes another $4-7$ years $[8,9]$. Still, it is estimated that only 1 formulation in 1000-2000 could eventually meet all the stringent requirements and progress into the market [9]. During the three phases of human trials, the safety, effectiveness, and side effects of candidate drugs are evaluated stage by stage [9]: In Phase I, the safety of the drugs and biologics are tested in less than 100 patients, which takes $1-2$ years and costs 50-150 million dollars, at $\sim 25 \%$ failure rate; in Phase II, the dosage ranges of the drugs and biologics are optimized with hundreds of patients, which takes 1-2 years and 100-200 million dollars at 25\% failure rate; in Phase III, the efficacy and side effects of the compounds are analyzed in up to thousands of patients, which requires 2-3 years and totals 150250 million dollars, at $\sim 35 \%$ of failure rate. Lastly, physicians, statisticians, chemists, pharmacologists and other scientists at the FDA will review the results of the clinical trial, prior to the final approval of the formulation, which takes around 6-10 months. Through this strict verification process, only a few nanomedicine formulations have been made commercially available to patients, despite the enormous amount of research on this subject from academic institutions.

Additionally, there are still significant concerns regarding clinical translation, as the properties and side effects of nanomedicine formulations are not yet fully understood. Since nanoparticles can cross the biological barriers, including cellular membranes as well as air-blood and blood-brain barriers in vivo, there are huge opportunities to access local regions where conventional materials cannot reach; however, this may adversely affect patients due to the potential for uncontrolled migration and deposition within tissues [10]. Moreover, the side effects of many new nanomedicine formulations cannot always be comprehensively measured during the clinical trial phase due to the small sample size of patients that does not completely represent the entire disease population.

Thus, it is necessary to rigorously test and assess nanomedicine formulations in preclinical models before proceeding to a clinical trial setting. For instance, in general medicine, serious adverse effects of drug therapies have been increasingly discovered after the clinical trial process was gradually expedited through legislative acts including the Prescription Drug User Fee Acts (PDUFA). Since the 1970s, approximately 35 drugs and biologics that were approved by the FDA have been recalled from the US market due to their fatal side effects that were unknown at the time of approval [11]. Therefore, it is imperative to enact strict and rigor- ous testing procedures for the premarket approval of nanomedicine in order to ensure patient safety.

\section{Advancing novel preclinical models}

Although there are several barriers to the clinical translation of nanomedicine formulations, it is imperative to seek novel and creative mechanisms to overcome these obstacles to allow commercial access to drugs that are more effective than those currently on the market. The implementation of novel preclinical testing models will enable these drugs to be rigorously tested and become available on the market at a faster pace. Animal models, already limited by existing economic and ethical burdens, are not optimal for this aim as they possess different genetic factors and thus do not accurately predict the human response to the drug. Therefore, we need more reliable in vitro model platforms, that can precisely mimic the properties and functions of the human organ systems to advance the clinical translation of nanomedicine [12].

Organ-on-a-chip systems are miniaturized human cell culture platforms that can mimic the microarchitecture, functions, and physiology of their in vivo counterparts, which can simulate pharmacokinetics/pharmacodynamics of drugs and thus accurately predict their efficacy and toxicity in the human body [12,13]. The human body contains a sophisticated network of blood vessels that interconnect organs; this circulatory system is essential in sustaining the functions of and communications among individual organs. In this integrated environment, neither organs nor their responses are isolated, meaning that the behavior of one organ upon treatment of a nanomedicine formulation will be contingent on that of the other linked organs. Therefore, building realistic in vitro human organ models embedded in an interconnected microfluidic platform will allow the observation of the drug's effect on each organ, and accurately predict drug efficacy and any potential side effects [12]. These models can be made across a range of complexity from either individual or multiple organ types, including the lung, kidney, blood vessel, airway, and bone marrow, among others [14], as well as their interconnected forms such as the micro cell culture analog $(\mu \mathrm{CCA})$ devices and the microphysiological systems where up to ten organs are integrated in order to represent their interactions $[15,16]$.

\section{Looking forward}

The field of nanomedicine is rapidly developing and, consequently, the demand for clinical translation is quickly expanding. Since rationally designed nanomaterials can lead to innovative treatment schemes and better patient outcomes, biomedical scientists and engineers should actively seek the best route to safely develop the field of 
nanomedicine. Biomimetic platforms, such as organson-a-chip, will provide unprecedented opportunities to accurately evaluate the safety and efficacy of nanomedicine formulations, leading to higher clinical trial success rates. Government organizations have recently allocated a significant amount of funding to help further the development of these innovative biomimetic platforms for drug delivery [17-20]. As interest in this field continues to grow, we envision that the clinical translation of nanomedicine will be significantly facilitated and will lead to groundbreaking revolutions in drug delivery platforms and ultimately, human healthcare.

\section{References}

1 Sun T, Zhang YS, Pang B et al. Engineered nanoparticles for drug delivery in cancer therapy. Angew. Chem. Int. Ed. Engl. 53, 12320-12364 (2014).

2 Irvine DJ, Hanson MC, Rakhra K et al. Synthetic nanoparticles for vaccines and immunotherapy. Chem. Rev. 115, 11109-11146 (2015)

3 Howes PD, Chandrawati R, Stevens MM. Colloidal nanoparticles as advanced biological sensors. Science 346, 1247390 (2014).

4 Jiang W, Kim BY, Rutka JT et al. Nanoparticle-mediated cellular response is size-dependent. Nat. Nanotechnol 3, 145-150 (2008).

5 Jang HL, Jin K, Lee J et al. Revisiting whitlockite, the second most abundant biomineral in bone: nanocrystal synthesis in physiologically relevant conditions and biocompatibility evaluation. ACS Nano 8, 634-641 (2013).

6 Jang HL, Zheng GB, Park J et al. In vitro and in vivo evaluation of whitlockite biocompatibility: comparative study with hydroxyapatite and $\beta$-tricalcium phosphate. $A d v$. Healthc. Mater. 5(1), 128-136 (2015).

7 Noorlander CW, Kooi MW, Oomen AG et al. Horizon scan of nanomedicinal products. Nanomedicine 10, 1599-1608 (2015).

8 Etheridge ML, Campbell SA, Erdman AG et al. The big picture on nanomedicine: the state of investigational and approved nanomedicine products. Nanomedicine 9, 1-14 (2013).

9 Pashuck ET, Stevens MM. Designing regenerative biomaterial therapies for the clinic. Sci. Transl. Med. 4, 160sr164-160sr164 (2012).

\section{Financial \& competing interests disclosure}

The authors gratefully acknowledge funding from the $\mathrm{Na}$ tional Institutes of Health (EB012597, AR057837, DE021468, HL099073, R56Al105024, AR068258, AR066193, EB022403, EB021148), and the Presidential Early Career Award for Scientists and Engineers (PECASE). The authors have no other relevant affiliations or financial involvement with any organization or entity with a financial interest in or financial conflict with the subject matter or materials discussed in the manuscript apart from those disclosed.

No writing assistance was utilized in the production of this manuscript.

10 Elsaesser A, Howard CV. Toxicology of nanoparticles. Adv. Drug Deliv. Rev. 64, 129-137 (2012).

11 Procon.Org. 35 FDA-approved prescription drugs later pulled from the market. http://prescriptiondrugs.procon.org

12 Zhang YS, Khademhosseini A. Seeking the right context for evaluating nanomedicine: from tissue models in petri dishes to microfluidic organs-on-a-chip. Nanomedicine 10, 685-688 (2015).

13 Bhise NS, Ribas J, Manoharan V et al. Organ-on-a-chip platforms for studying drug delivery systems. J. Control. Release 190, 82-93 (2014).

14 Huh D, Hamilton GA, Ingber DE. From 3D cell culture to organs-on-chips. Trends Cell Biol. 21, 745-754 (2011).

15 Esch M, King T, Shuler M. The role of body-on-a-chip devices in drug and toxicity studies. Annu. Rev. Biomed. Eng. 13, 55-72 (2011).

16 Wikswo JP. The relevance and potential roles of microphysiological systems in biology and medicine. Exp. Biol. Med. 239, 1061-1072 (2014).

17 News M. DARPA and NIH to fund 'human body on a chip' research. http://news.mit.edu

18 Center WFBM. Wake Forest Baptist leads \$24 million project to develop "Body on a Chip". www.wakehealth.edu

19 Laboratory LaN. ATHENA desktop human "body" could reduce need for animal drug tests. www.lanl.gov

20 Health NIO. Tissue Chip Projects (2014). www.ncats.nih.gov 